\title{
Tuning of Metal/Insulator transition around room temperature of perovskites $\mathrm{Sm}_{1-\mathrm{x}} \mathrm{Nd}_{\mathbf{x}} \mathrm{NiO}_{3}$
}

\author{
G. Frand ${ }^{1}$, O. Bohnke ${ }^{2}$, P. Lacorre, and J. L. Fourquet, \\ Laboratoire des Fluorures (URA 449 CNRS), Université du Maine, 72017 Le Mans Cedex, France \\ A. Carré and B. Eid \\ Corning Europe Incorporated, 7 bis, avenue de Valvins, 77210 Avon, France \\ J. G. Théobald and A. Gire \\ Laboratoire de Spectroscopie Hertzienne et d'Electronique, Université de Franche-Comté, 25030 Besançon \\ Cedex, France
}

\begin{abstract}
We report here a study of the synthesis and properties of the solid solution series $\mathrm{Sm}_{1-\mathrm{x}} \mathrm{Nd}_{\mathrm{x}} \mathrm{NiO}_{3}$. It was found to crystallize in the $\mathrm{GdFeO}_{3}$-type orthorhombically distorted perovskite structure, The metal-insulator $(\mathrm{M} / \mathrm{I})$ transition was mapped out as a function of the composition, It is possible to control this transition between 200 and $410 \mathrm{~K}$ by simply adjusting the composition. For instance, the MII transition occurs at room temperature for $\mathrm{Sm}_{0.55} \mathrm{Nd}_{0.45} \mathrm{NiO}_{3}$, and the variation of the transition temperature with composition is about $2.3 \mathrm{~K} / \mathrm{x} \%$. These phase transitions have been studied by differential scanning calorimetry, microwave procedure, and $\mathrm{X}$-ray diffraction. Microwave data reveal hysteresis effects, An interpretation of the experimental results may be the coexistence of the metallic and insulating phases. On the other hand, we have observed that the enthalpy change of phase transition decreases when the powder is ground. This phenomenon can be related to amorphization of the material.
\end{abstract}

\section{INTRODUCTION}

Perhaps the most interesting property of orthorhombically distorted perovskite oxides of the general formula $R \mathrm{NiO}_{3}(R=$ rare earth) is their insulator-metal transition (1). The transition temperature strongly decreases with increasing size of the rare-earth ion; for instance, it has been observed in $\mathrm{NdNiO}_{3}$ and $\mathrm{SmNiO}_{3}$ at 200 and $410 \mathrm{~K}$, respectively $(1,2)$. A small discontinuous decrease in the unit-cell volume is associated with the insulator-metal transition. This contraction has been attributed to the onset of electronic delocalization in the metallic state (3). A magnetic transition has also been observed in these compounds which is correlated to the M/I transition for $R=$ Pr and Nd, but not for the other members of the series. A study of $\mathrm{Nd}_{1-x} \mathrm{La}_{x} \mathrm{NiO}_{3-\delta}$ series (4) shows that the substitution of $\mathrm{Nd}$ by $\mathrm{La}$ in the $\mathrm{NdNiO}_{3}$ system induces several effects on crystallographic, magnetic, and electrical properties. In this paper, we report the preparation and a study of the insulator-metal properties of the solid solution $\mathrm{Sm}_{1-x} \mathrm{Nd}_{x} \mathrm{NiO}_{3}$. We show that it is possible to adjust the $\mathrm{M} / \mathrm{I}$ transition around room temperature by appropriately choosing $x$.

\footnotetext{
'Doctoral Fellowship (CIFRE) from Corning Europe Inc., 7 bis, Avenue de Valvins, 77210 Avon, France.

${ }^{2}$ To whom correspondence should be addressed.
}

\section{EXPERIMENTAL}

\section{Sample Preparation}

The $\mathrm{Sm}_{1-x} \mathrm{Nd}_{x} \mathrm{NiO}_{3}$ powders were prepared by synthesis under high oxygen pressure. The preparation of the $\mathrm{Sm}_{1-x} \mathrm{Nd}_{x} \mathrm{NiO}_{3}$ series involves the following reaction:

$$
\begin{aligned}
(1-x) \mathrm{Sm}_{2} \mathrm{O}_{3} & +x \mathrm{Nd}_{2} \mathrm{O}_{3}+2 \mathrm{NiO} \\
+1 / 2 \mathrm{O}_{2} & \rightarrow 2 \mathrm{Sm}_{1-x} \mathrm{Nd}_{x} \mathrm{NiO}_{3} .
\end{aligned}
$$

The samples were synthesized from an intimate mixture of oxides obtained from the decomposition of the corresponding nitrates. The nitrates were obtained by dissolving a stoichiometric mixture of the elementary oxides in concentrated nitric acid and then drying the solution. After decomposition of the residue at $300^{\circ} \mathrm{C}$, this intimate mix- 


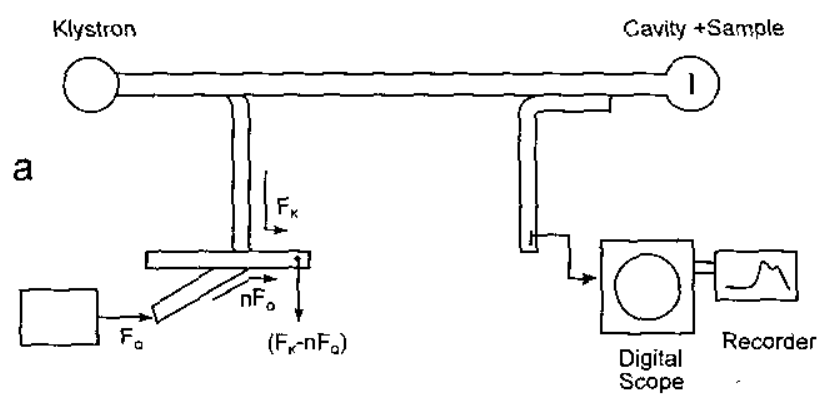

b

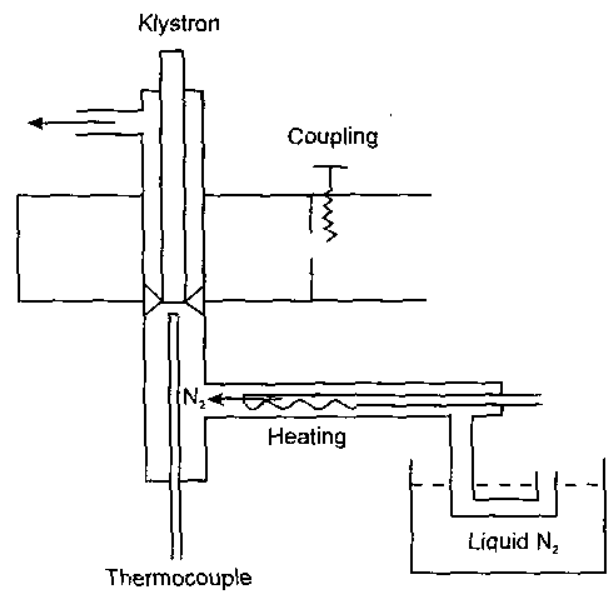

FIG. 1. Schematic diagrams of (a) microwave setup with cavity and (b) setting temperature sample.

ture of oxides was fired at $700^{\circ} \mathrm{C}$ for several hours and pressed into small pellets. These pellets of synthesized powders were fired at $1000^{\circ} \mathrm{C}$ for several days under $150-$ 160 bars of oxygen with several intermediate regrindings and repressings. The resulting samples were black in color.

Preparations were carried out for the $x=0.1,0.25,0.35$, $0.425,0.45,0.5,0.6,0.75$, and 0.9 members of the series.

\section{DSC Measurements}

DSC measurements were made on a Perkin-Elmer DSC4 apparatus in order to determine the phase transition temperature for all the samples. In order to decrease the size of the particles the powder was ground. DSC measurements were made on a Dupont DSC 9900 system to study the effect of the grinding time on the intensity of the endothermic peak corresponding to their $\mathrm{I} \rightarrow \mathrm{M}$ transition. In the first case, samples weighing $35-50 \mathrm{mg}$, sealed in aluminium crucibles, were heated from -100 to $200^{\circ} \mathrm{C}$; in the second case, samples weighing $\sim 20 \mathrm{mg}$, sealed in aluminium crucibles, were heated from -30 to $200^{\circ} \mathrm{C}$. For both measurements, heating rate of $20^{\circ} \mathrm{C} / \mathrm{min}$ was applied, and an empty aluminium crucible was used as reference.

\section{Microwave Procedure}

Microwave techniques were developed at the Laboratoire de spectroscopie hertzienne et d'electronique (Besançon). Microwave measurements were performed on samples enclosed in sealed quartz tubes, inserted in a microwave cavity as shown in Fig. 1a.

The presence of a sample in the cavity leads to:

-A resonance frequency shift according to the relationship

$$
\underline{\Delta f}=-\frac{1}{2} \eta \chi^{\prime}
$$

where $\chi^{\prime}$ is the real part of the microwave susceptibility $\left(\chi^{\prime}=\varepsilon_{r}-1\right)$ and $\eta$ is the filling coefficient:

$$
\eta=\frac{\iiint_{\text {sample }} E^{2} d V}{\iiint_{\text {cavity }} E^{2} d V}
$$

-A variation of the quality factor $Q$ :

$$
\Delta(1 / Q)=\eta \chi^{\prime \prime} .
$$

$1 / Q$ is obtained from the linewidth $\Delta F$ of the resonant cavity $(1 / Q=\Delta F / A$ ). If $\alpha$ is the coupling factor, the quality factor $Q$ of the coupled cavity of the waveguide is related to the factor $Q_{0}$ (quality factor of the empty cavity) by $Q_{0}=Q(1+\alpha)$. By using an impedance adapter, the power

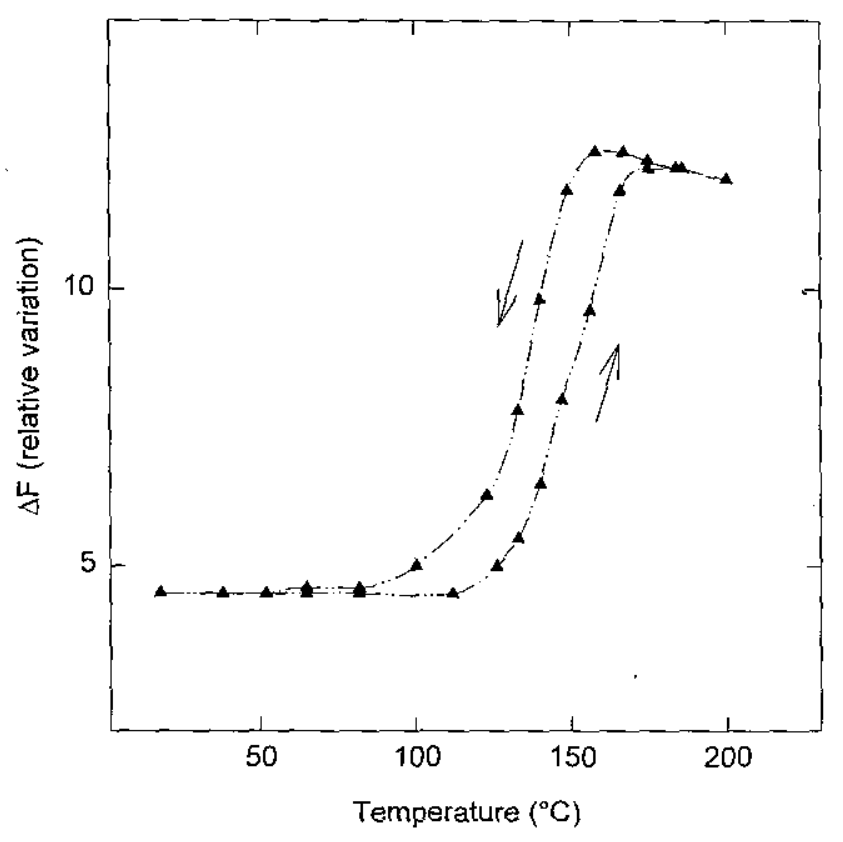

FIG. 2. Plot or resonance frequency linewidth $\Delta F$ versus temperature for a $\mathrm{Sm}_{0.9} \mathrm{Nd}_{0,1} \mathrm{NiO}_{3}$ composition. 


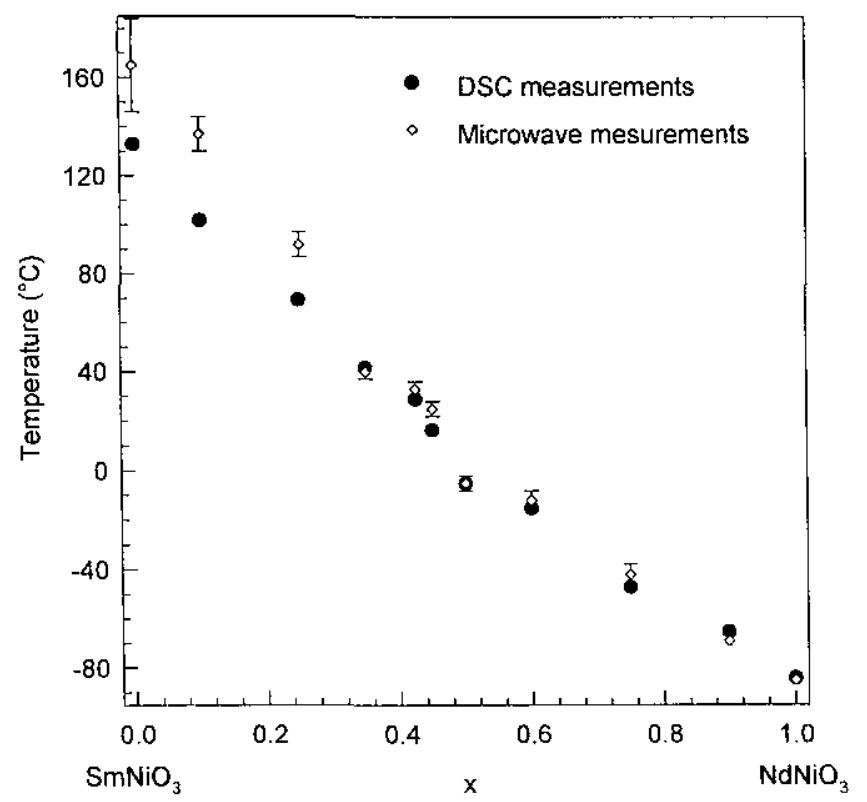

FIG. 3. Transition temperature versus composition $x$ for $\mathrm{Sm}_{1-x} \mathrm{Nd}_{x} \mathrm{NiO}_{3}$.

given by the klystron is totally absorbed by the cavity at the resonance. Then, $\alpha=1$, and the measurement is simplified. The cavity frequency is $9192 \mathrm{MHz}$. The microwave susceptibility $\left(\chi=\chi^{\prime}-j \chi^{\prime \prime}\right)$ has an equivalence in conductibility $\left(\sigma=\sigma^{\prime}+j \sigma^{\prime \prime}\right)$ by the relationship $\sigma=$ $j \omega \varepsilon_{0} \chi$. In the experiments described here, $\Delta / f$ is small. Therefore, the variations of the quality factor $Q$ are linked to the conductibility by the relationship $\sigma=\omega \varepsilon_{0} \chi^{\prime \prime}$. The variation of the linewidth $\Delta F$ is proportional to the conductibility variation.

The setting temperature of the sample is obtained by a nitrogen stream as shown in Fig. 1b. The temperature stability during the measurements is $\pm 1-2 \mathrm{~K}$.

The cavity resonance curve is recorded with a digital oscilloscope. The measurement precision is directly linked to the precision at which the frequencies are measured. By using the harmonics of a reference oscillator, a precision of $10^{-2} \mathrm{MHz}$ is obtained.

\section{$X-$ Ray Diffraction}

Powder X-ray diffraction patterns were collected at room temperature on a D500 Siemens diffractometer using $\mathrm{Cu} K \alpha$ radiation. Diffraction patterns were recorded in the $2 \theta$ range $20^{\circ}-130^{\circ}$ (step $\Delta 2 \theta=0.02^{\circ}-0.03^{\circ}$ ). In order to reduce preferred orientation, the samples were dusted through a sieve on the holder surface.

All X-ray data analysis are performed with a modified version (5) of the Rietveld method (6). Lines shapes are approximated by a pseudo-Voigt function.

\section{RESULTS AND DISCUSSION}

\section{Transition Phase Behavior}

Figure 2 shows the dependence of the linewidth $\Delta F$ of the resonance frequency obtained by microwave measurements as a function of temperature. These data are recorded in a heating-up followed by a cooling-down process. A phase transition is clearly observed. At low temperature, the linewidth $\Delta F$ of the resonance frequency is constant: the compound is an insulator. The sudden increase of $\Delta F$ coincides with the conductibility appearance. After the transition temperature, $\Delta F$ slowly decreases meaning that conductibility decreases. This is characteristic of a metallic behavior. Such a metal-insulator transition is found for every composition in the series. A temperature hysteresis is observed in the cooling-down measurements. The hysteresis temperature interval depends on the solid solution composition: the lower the $x$ value (solid solution rich in $\mathrm{Sm}$ ), the higher the hysteresis temperature interval. The phase transition temperatures shown in Fig. 3 are taken in the middle of the hysteretic temperature range; the error bar is the width of the hysteresis. As we can see in the cooling-down curve, the metallic region extends below the temperature found in the heating-up process. At still lower temperatures, the semiconductorlike behavior is reached. Hysteresis around the metal-insulator transition have been clearly observed elsewhere on $\mathrm{PrNiO}_{3}$ (7) and $\mathrm{NdNiO}_{3}(8)$ from resistance measurements. In our case, we could suppose that the metallic phase coexists with the semiconducting one over a wide temperature interval close to the metal-insulator transition. This blend of two components has an overall resistivity which is determined by the relative volume of both phases and by the shape and

TABLE 1

Solid Solution Enthalpy Changes Obtained from Perkin Elmer DSC Measurements

\begin{tabular}{lc}
\hline $\begin{array}{c}\text { Samples } \\
x \text { composition }\end{array}$ & $\begin{array}{c}\text { Endothermic DSC peak } \\
(\mathrm{J} / \mathrm{g})\end{array}$ \\
\hline 0 & 2.4 \\
0.10 & 2.1 \\
0.25 & 1.5 \\
0.35 & 1.4 \\
0.425 & 1.4 \\
0.45 & 1.3 \\
0.50 & 0.8 \\
0.60 & 0.8 \\
0.75 & 1.6 \\
0.90 & 1.0 \\
1 & 1.1 \\
\hline
\end{tabular}




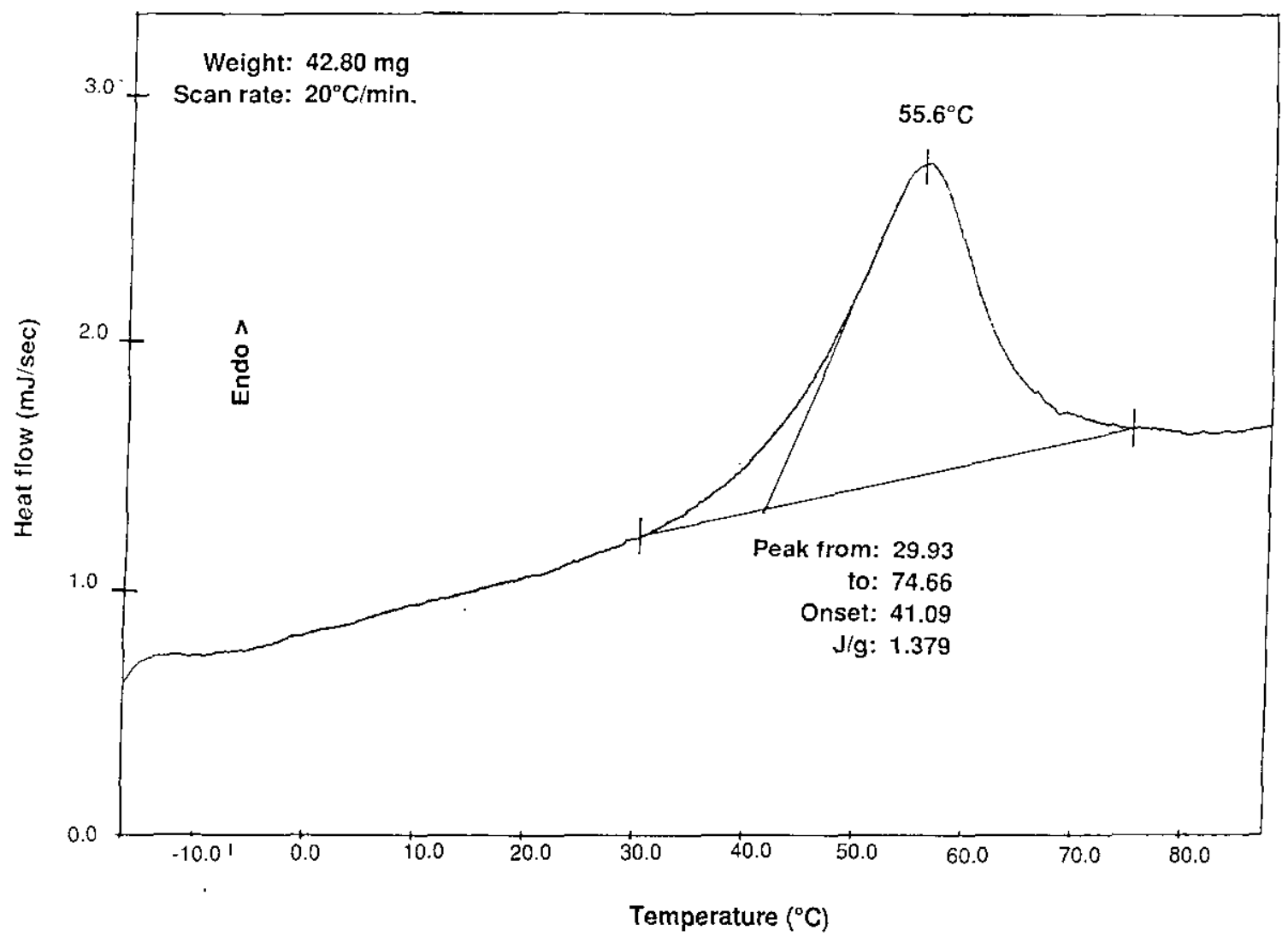

FIG. 4. DSC curve for the $\mathrm{Sm}_{0.65} \mathrm{Nd}_{0.35} \mathrm{NiO}_{3}$ composition. The endothermic peak corresponds to the metal-insulator phase transition.

distribution of the particles or domains of each phase (9). This model, explained elsewhere (7), can be used to describe the resistivity variations across the metallic to insulator transition. Sample conductibility is proportional to the variation in $\Delta F$. Metallic conductivities calculated from microwave measurements are found to be around $3.10^{5}$ $\mathrm{S} / \mathrm{m}$. This value is in good agreement with the conductivity of $\mathrm{PrNiO}_{3}$, i.e., $2.5 .10^{5} \mathrm{~S} / \mathrm{m}(10)$. The temperature of the metal-insulator transition is observed to increase as expected from $200 \mathrm{~K}\left(\mathrm{NdNiO}_{3}\right)$ to $400 \mathrm{~K}\left(\mathrm{SmNiO}_{3}\right)$.

Differential scanning calorimetry is another method used to measure the insulator-metal transition temperature of all compositions. A typical plot is given in Fig. 4. We have only made DSC measurements during the heating sweep. The transition temperatures are determined from the onset of the endothermic DSC peak. The temperature range of the transition process is large $\left(\sim 40^{\circ} \mathrm{C}\right)$. In DSC experiments, transitions are shown to be clearly first order transitions. The transition temperatures thus found are in good agreement with those obtained from microwave measurements as shown in Fig. 3. Endothermic peak area calculation leads to the reaction thermal content which is approximately the enthalpy change. A calculation error is made on the endothermic peak area determination due to an approximate baseline of the DSC measurements. Enthalpy changes are shown in Table 1.

These values are in good agreement with those found elsewhere (approximately 1.6 and $2.0 \mathrm{~J} / \mathrm{g}$ for $\mathrm{Nd}$ and $\mathrm{Sm}$ pure compound, respectively) (1). It seems that there is no relationship between composition and enthalpy change.

\section{Grinding Time Effect on Endothermic DSC}

\section{Peak Intensity}

In order to obtain a given composition with different sizes of particles, we used an agate grinder with agate marbles to grind the corresponding powder. Grinding time was the chosen parameter. The composition we ground at different times was $\mathrm{Sm}_{0.575} \mathrm{Nd}_{0.425} \mathrm{NiO}_{3}$. Then, by DSC measurements, the endothermic peak area corresponding to the heat content of the transition for each ground powder was determined. Grinding times and endothermic DSC peak areas are listed in Table 2.

From Table 2 it can be observed that the endothermic DSC peak area becomes smaller as the grinding time increases. Moreover for grinding time longer than $60 \mathrm{~min}$ both the DSC and the microwave measurements clearly show that the phase transition disappears. The disappear- 
TABLE 2

DSC Peak Area for $\mathrm{Sm}_{0.575} \mathrm{Nd}_{0.425} \mathrm{NiO}_{3}$ Obtained after Different Grinding Times

\begin{tabular}{cc}
\hline $\begin{array}{c}\text { Grinding time } \\
(\mathrm{min})\end{array}$ & $\begin{array}{c}\text { Endothermic DSC peak area } \\
(\mathrm{J} / \mathrm{g})\end{array}$ \\
\hline 0 & 1.4 \\
1 & 1.2 \\
2 & 1.0 \\
10 & 0.8 \\
20 & 0.4 \\
30 & 0.2 \\
40 & 0.1 \\
60 & no peak \\
\hline
\end{tabular}

ance of the phase transition is certainly linked to an amorphization process of the material. This hypothesis is corroborated by the nearly flat X-ray diffraction pattern obtained with a powder ground for $5 \mathrm{hr}$ and by the progressive broadening of diffraction peaks of the ground powder. So, we can suppose that phase transition takes place in perfectly crystallized material. The quantity of amorphous material increases as the grinding time increases, and consequently, the quantity of crystallized material decreases; DSC measurements show a weaker and weaker endothermic peak. The material with phase transition, isolated in an amorphous environment, could give some broad diffraction peaks and the phase transition would no longer be measurable. For longer times of grinding, material is completely amorphous, so there is no phase transition and no $\mathrm{X}$-ray diffraction. The transition is restored only by an annealing of the finely ground powder in synthesis conditions. An annealing at moderate temperature (e.g., $400^{\circ} \mathrm{C}$ for $16 \mathrm{hr}$ ) is not able to restore the transition; the diffraction spectrum shows that the sample remains largely amorphous. Indeed, the annealing in synthesis conditions induces the growth of crystallized grains which consequently leads to the reappearance of the phase transition. The enthalpy change of the annealed material is found to be $0.7 \mathrm{~J} / \mathrm{g}$.

\section{Room-Temperature Cell Parameters of $\mathrm{Sm}_{1-x} \mathrm{Nd}_{x} \mathrm{NiO}_{3}$}

The X-ray powder diffraction patterns of $\mathrm{Sm}_{1-x} \mathrm{Nd}_{x} \mathrm{NiO}_{3}$ solid solution are indexed in the same space group $(\mathrm{Pbnm})$
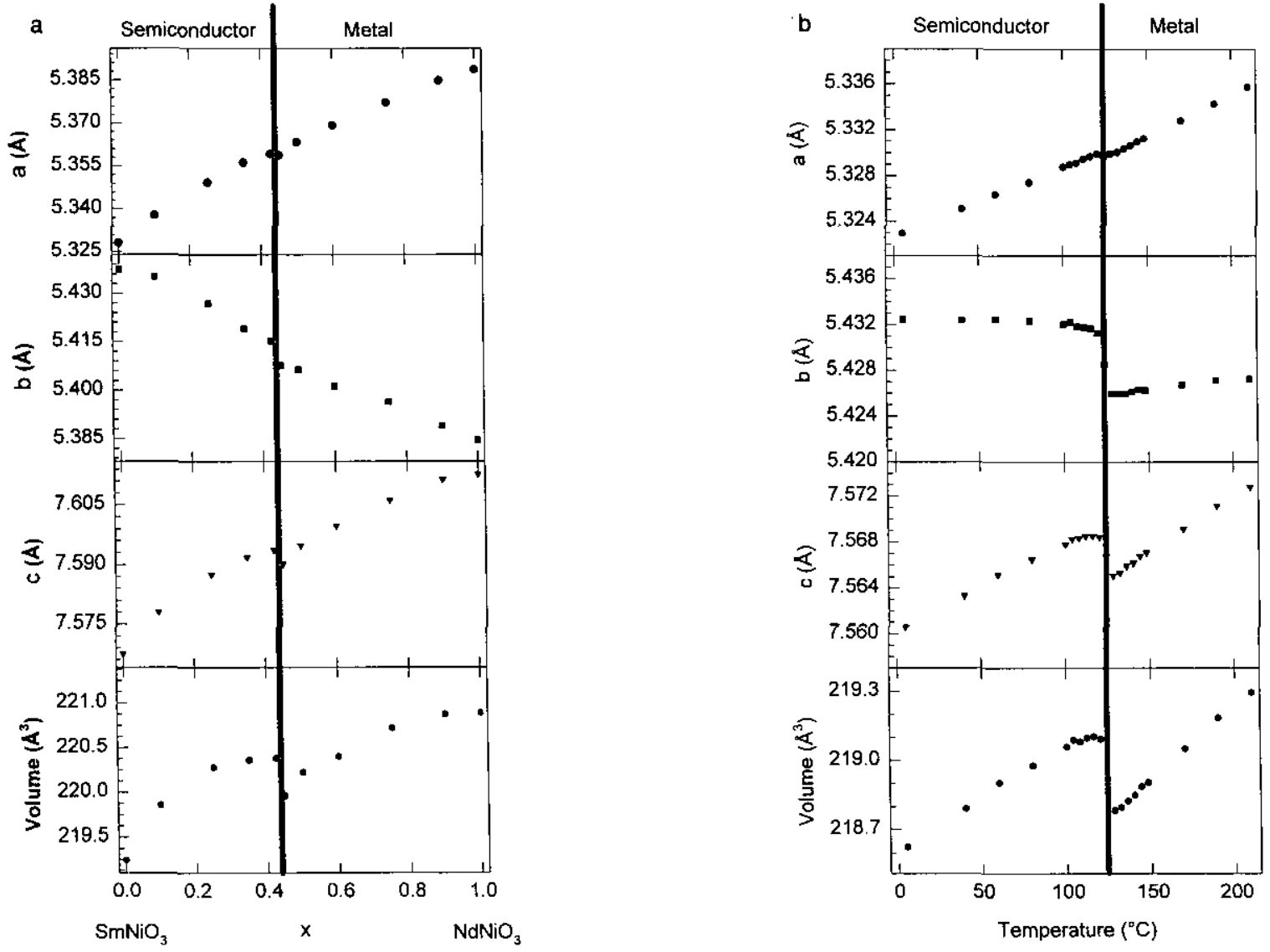

FIG. 5. (a) Composition dependence of solid solution lattice constants. Data obtained by X-ray diffraction at room temperature (this work). (b) Thermal evolution of $\mathrm{SmNiO}_{3}$ lattice constants (X-ray diffraction, from Ref. (3)). In both cases, a similar anomaly occurs at the metal-insulator transition (grey line). 


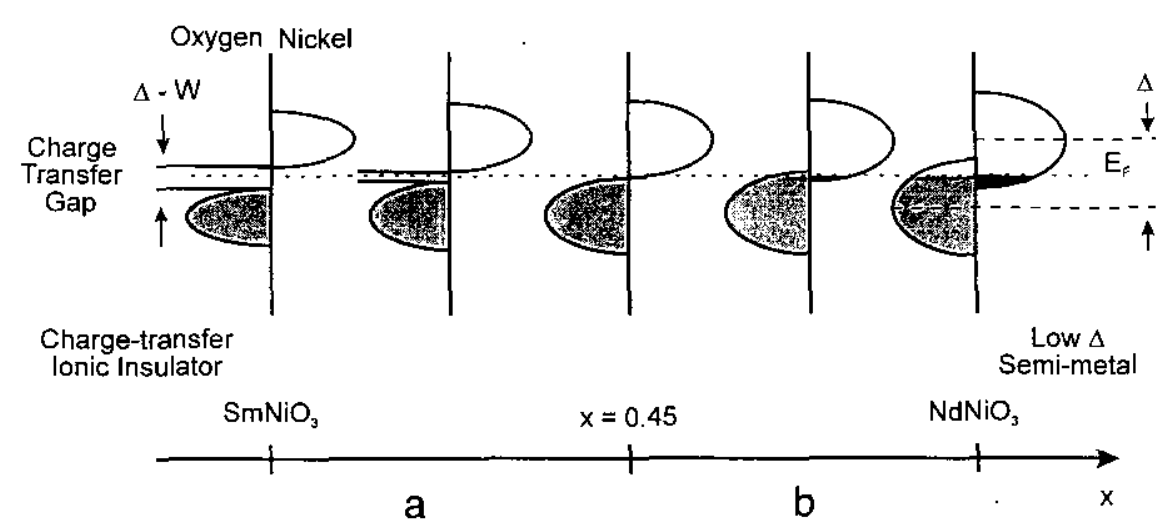

FIG. 6. Schematic band diagram, at room temperature for $\mathrm{Sm}_{1-x} \mathrm{Nd}_{x} \mathrm{NiO}_{3}$ (adapted from (10)): (a) insulator and (b) semimetal.

as $\mathrm{GdFeO}_{3}(11)$. The resulting lattice constants are plotted as a function of composition in Fig. 5a. The evolution of the refined lattice parameters from $\mathrm{SmNiO}_{3}$ to $\mathrm{NdNiO}_{3}$ exhibits a small discontinuity. This behavior can be explained as follows. X-ray powder diffraction patterns were recorded at room temperature and transition temperatures found by DSC and microwave measurements for $\mathrm{Sm}_{1-x} \mathrm{Nd}_{x} \mathrm{NiO}_{3}$ solid solutions take place between $200 \mathrm{~K}$ $\left(\mathrm{NdNiO}_{3}\right)$ and $400 \mathrm{~K}\left(\mathrm{SmNiO}_{3}\right)$, that is below and above room temperature. The break we observe on lattice parameter curves corresponds to the solid solution composition which has its transition temperature near the value at which the X-ray diffraction pattern temperature is recorded (i.e., room temperature $\sim 25^{\circ} \mathrm{C}$ ). Thus, all the samples with $x>$ 0.45 are in a metallic state (their transition temperatures $T_{\mathrm{MI}}$ are lower than room temperature), while all compositions with $x<0.45$ are in a semiconductor state. The lattice parameter variations are a manifestation of subtle changes in the structural arrangement between the metallic and semiconducting phases. The composition dependence of lattice constants can be compared to the thermal evolution of $\mathrm{SmNiO}_{3}$ cell parameters reported previously (3) (Fig. 5b). In pure $\mathrm{SmNiO}_{3}$, the cell parameters undergo an abrupt change at the transition temperature. All three cell constants decrease abruptly when the compound becomes metallic. The cell volume undergoes a small decrease due to a slight decrease of $\mathrm{Ni}-\mathrm{O}$ distances. Oxygen displacements upon decreasing temperature through the transition are merely a regular accommodation of the perovskite framework to the abrupt slight enhancement of the $\mathrm{Ni}-\mathrm{O}$ distance, which itself is triggered by the electronic localization. Figure 5 seems to show that, at least for $\mathrm{SmNiO}_{3}$ and in first approximation, a parallel can be made between thermal expansion and substitution of $\mathrm{Sm}$ by a larger rare earth.

In these compounds, the occurrence of the metallic regime is thought to originate from the closing of a charge transfer gap between the occupied oxygen $2 p$ valence band and the unoccupied $\mathrm{Ni} 3 d$ conduction band (12). The closing of the gap could be controlled by the bandwidth, which increases with the $\mathrm{Ni}-\mathrm{O}-\mathrm{Ni}$ angle. This angle directly depends on the size of the rare earth, increasing with $x$. Because of the low scattering power of oxygen for X-rays relative to $\mathrm{Sm}$ or $\mathrm{Nd}$, no reliable information could be obtained on the $\mathrm{Ni}-\mathrm{O}-\mathrm{Ni}$ angle and $\mathrm{Ni}-\mathrm{O}$ distance in solid solution $\mathrm{Sm}_{1-x} \mathrm{Nd}_{x} \mathrm{NiO}_{3}$. However, it is well shown by the neutron-diffraction study of $\mathrm{RNiO}_{3}$ made by GarciaMunoz et al. (3) that the $\mathrm{Ni}-\mathrm{O}-\mathrm{Ni}$ angle increases with rare earth size, from $\mathrm{NdNiO}_{3}$ to $\mathrm{PrNiO}_{3}$. The model of charge-transfer gap for insulating and metallic phases proposed by Torrance et al. (12) can be used for the solid solutions $\mathrm{Sm}_{1-x} \mathrm{Nd}_{x} \mathrm{NiO}_{3}$; a schematic diagram is shown in Fig. 6 . For $x<0.45$, the compounds are insulating at room temperature, the $\mathrm{Ni}-\mathrm{O}-\mathrm{Ni}$ angle is small, and the valence and conduction bands do not overlap (Fig. 6a). As $x$ increases (i.e., the $\mathrm{Nd}$ composition increases), the bandwidth increases, so the charge-transfer gap decreases until the valence and conduction bands overlap, giving rise to a semimetallic state (Fig. 6b).

\section{CONCLUSION}

The behavior of the metal-insulator transition of $\mathrm{Sm}_{1-x} \mathrm{Nd}_{x} \mathrm{NiO}_{3}$ compounds have been explained in the light of what is already known about the $\mathrm{RNiO}_{3}$ series. The phase transitions have been detected by microwave and DSC measurements. Correlating the structural and transition measurements, we show that increasing the Nd content in $\mathrm{Sm}_{1-x} \mathrm{Nd}_{x} \mathrm{NiO}_{3}$ leads to a decrease in the transition temperature. It is possible to finely tune the insulatorto-metal transition temperature around room temperature by controlling the $\mathrm{Nd}$ content, which determines the $\mathrm{Ni}-$ $\mathrm{O}-\mathrm{Ni}$ angle and thus the band overlap. The composition which undergoes a transition at room temperature is $\mathrm{Sm}_{0.55} \mathrm{Nd}_{0.45} \mathrm{NiO}_{3}$. 


\section{REFERENCES}

1. P. Lacorre, J. B. Torrance, J. Pannetier, A. I. Nazzal, P. W. Wang, T. C. Huang, and R. L. Siemens, J. Solid State Chem. 91, 225 (1991).

2. J. K. Vassiliou, M. Hornbostel, R. Ziebarth, and F. J. DiSalvo, J. Solid State Chem. 81, 208 (1989).

3. J. L. Garcia-Munoz, J. Rodriguez-Carvajal, P. Lacorre, and J. B. Torrance, Phys. Rev. B 46(8), 4414 (1992).

4. J. Blasco and J. Garcia, J. Phys. Condens Matter 6, 10759 (1994).

5. J. Rodriguez-Carvajal, program FullProf, private communication.

6. H. M. Rietveld, J. Appl. Crystallogr. 2, 65 (1969).
7. X. Granados, J. Fontcuberta, X. Obradors, and J. B. Torrance, Phys. Rev. B 46(24), 15683 (1992).

8. X. Granados, J. Fontcuberta, X. Obradors, Ll. Manosa, and J. B. Torrance, Phys. Rev. B 48(16), 11666 (1993).

9. P. L. Rossiter, in "The Electrical Resistivity of Metals and Alloys" (R. W. Cahn, E. A. Davis, and I. M. Ward, Eds.), Cambridge Solid Statc Science Series. Cambridge Univ. Press, Cambridge, 1991.

10. X. Q. Xu, J. L. Peng, Z. Y. Li, H. L. Ju, and R. L. Greene, Phys. Rev. B 48(2), 1112 (1993).

11. S. Geller, J. Chem. Phys. 24, 1236 (1956).

12. J. B. Torrance, P. Lacorre, A. I. Nazzal, E. J. Ansaldo, and Ch. Niedermayer, Phys. Rev. B 45(14), 8209 (1992). 